\title{
Cooperativity during Melting and Molecular Exchange in Micelles with Crystalline Cores
}

\author{
Nico König, ${ }^{1,2,}$ Lutz Willner, ${ }^{1, *}$ Vitaliy Pipich, ${ }^{3, \uparrow}$ Thomas Zinn, ${ }^{2, \dagger}$ and Reidar Lund ${ }^{2, \$}$ \\ ${ }^{1} J u ̈ l i c h$ Centre for Neutron Science (JCNS) and Institute for Complex Systems (ICS), \\ Forschungszentrum Jülich GmbH, 52425 Jülich, Germany \\ ${ }^{2}$ Department of Chemistry, University of Oslo, P.O. Box 1033, Blindern, 0315 Oslo, Norway \\ ${ }^{3}$ Jülich Centre for Neutron Science (JCNS) at Heinz, Maier-Leibnitz. Zentrum (MLZ), \\ Forschungszentrum Jülich GmbH, 85747 Garching, Germany
}

(Received 25 July 2018; revised manuscript received 19 November 2018; published 21 February 2019)

\begin{abstract}
Molecular exchange processes are important equilibration and transport mechanisms in both synthetic and biological self-assembled systems such as micelles, vesicles, and membranes. Still, these processes are not entirely understood, in particular the effect of crystallinity and the interplay between cooperative melting processes and chain exchange. Here we focus on a set of simple polymer micelles formed by binary mixtures of poly(ethylene oxide)-mono- $n$-alkyl-ethers $\left(\mathrm{C}_{n}\right.$-PEO5) which allows the melting point to be tuned over a wide range. We show that the melting transition is cooperative in the confined 4-5 nm micellar core, whereas the exchange process is widely decoupled and unimeric in nature. As confirmed by differential scanning calorimetry, the total activation energy for ejecting a molecule out of the micellar core below the melting point is the sum of the enthalpy of fusion and the corresponding activation energy in the melt state. This suggests that a "local, single-chain melting process" preludes the molecular diffusion out of the micelle during chain exchange.
\end{abstract}

DOI: 10.1103/PhysRevLett.122.078001

Partially crystalline, self-assembling systems with multiple components are omnipresent in nature with living cells as a prominent example. Their structural integrity is maintained by a membrane consisting of various lipids that can be in a liquid, gel, or crystalline state. For other self-assembling systems like block copolymers with crystallizable blocks, crystallization may even be the driving force behind self-assembly and can yield a whole range of remarkable nanostructural morphologies [1]. In the resulting micellar systems with crystalline cores of typically a few nanometers in diameter, interesting questions relate to the role of confinement. For example, it was recently discovered that the melting points of $n$-alkanes under soft nanoscopic confinement in micellar cores exhibit a simple Gibbs-Thomson behavior [2], typical for hard-confined liquids. In this context, it is also interesting how the dynamics of self-assembling systems, like molecular exchange kinetics, are affected by confinement and crystallinity, as these processes control the equilibration and sometimes even the resulting morphology.

So far, the existing theories for micelle kinetics have been devoted to amorphous micelles. For instance, the general theory developed by Halperin and Alexander [3] considers starlike micelles with molten cores where the chain expulsion is the rate-determining step. The expulsion is pictured as a process in which the chain diffuses over a free energy barrier with an activation energy $E_{a}$, which stems from the additional solvophobic surface created upon chain expulsion. This is a first-order kinetic process where the fraction of exchanged chains is described by an exponential relaxation function $R(t)=\exp (-t / \tau)$. Here the characteristic timescale is given by $\tau=\tau_{0} \exp \left[E_{a} /\left(k_{B} T\right)\right]$, where $k_{B}$ is Boltzmann's constant, $T$ the absolute temperature, and $\tau_{0}$ a prefactor that is associated with the attempt time, i.e., the time between two consecutive attempts of a molecule to leave the micellar core. In a previous study of $\mathrm{C}_{n}$-PEO5 micelles [4], we could show that in molten cores the activation energy $E_{a}$ scales linearly with the length of the solvophobic block $n$ because the relatively short alkyl block does not fully collapse into a spherical shape-in contrast to the $E_{a} \propto n^{2 / 3}$ scaling of fully collapsed solvophobic blocks predicted by Halperin and Alexander [3]. Since $R(t)$ is an exponential function of $\tau$ and the exchange time $\tau$ in turn depends exponentially on $E_{a} \propto n$, the relaxation function shows an extremely strong sensitivity to the solvophobic block length. For block copolymers with even a narrow distribution of $n$ (dispersity $M_{w} / M_{n}$ close to unity), there is thus a relatively broad distribution of exchange rates which results in a close-to-logarithmic decay instead of a single exponential [5-7]. This renders an exact determination of $\tau$ and $E_{a}$ impossible for block copolymers with finite dispersity. The effect is even more drastic in binary block copolymer mixtures of different molecular weight, so only qualitative conclusions can be drawn $[8,9]$. In this work, our goal is twofold: (i) understand the role of crystallinity and possible effects of cooperativity quantitatively and (ii) understand the mutual 
influence on the dynamics in systems with multiple components.

Until now, it has been found that in micellar systems crystallinity yields an additional energy barrier upon chain expulsion $[4,10]$, but the interplay between the cooperative melting processes and chain exchange is still unknown. An interesting approach to shed light on these processes is coassembling different amphiphiles which allows the melting point to be tuned. Here we use binary mixtures of amphiphilic, micelle-forming copolymers as a model system, namely, two poly(ethylene oxide)-mono- $n$-alkylethers $\left(\mathrm{C}_{n}\right.$-PEO5 with different alkyl lengths $n=22,28$ and a PEO molecular weight of $5 \mathrm{~kg} / \mathrm{mol}$ ) in aqueous solution. The polymers are prepared by well-established living anionic polymerization techniques leading to materials with desired PEO molar mass and narrow molar mass distributions of $M_{w} / M_{n}<1.04$ [11]. The $n$-alkanes, on the other hand, are monodisperse, $M_{w} / M_{n}=1$, which assures a single expulsion time, and do not exhibit the strong broadening inherent in polydisperse systems. Calorimetry and densiometry data show that the hydrophobic blocks mix within the micellar cores, and the melting point can be tuned continuously from 29 to $57^{\circ} \mathrm{C}$. In contrast, neutron scattering measurements reveal that the molecular exchange kinetics is governed by two unique expulsion rates corresponding to each component, both in the molten and crystalline state. That means, while the individual diffusion processes are decoupled, the two components are still subject to the same cooperative melting process which, below the melting point, imposes an additional activation energy term equal to the enthalpy of fusion $\Delta H_{\text {fus }}$.

The micelles were structurally characterized by smallangle neutron and $\mathrm{x}$-ray scattering (SANS, SAXS). Figure 1(a) shows representative SANS data of neat $\mathrm{C}_{28}$ and $\mathrm{C}_{22}$-PEO5 solutions and of a solution of a premixed $1: 1$ blend. A fit analysis of the scattering data using a
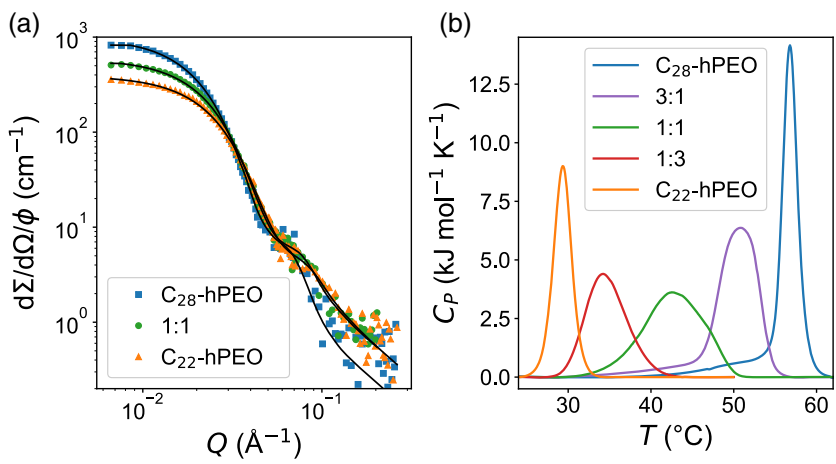

FIG. 1. (a) SANS data of pure $\mathrm{C}_{28^{-}}$and $\mathrm{C}_{22}-h$ PEO5 micellar solutions and of a premixed 1:1 blend (volume fraction $\phi=0.25 \mathrm{vol} \%$ ). Solid lines represent fits according to a quantitative core-shell model reported in Ref. [11] and outlined in the Supplemental Material [12]. The key fit parameters are shown in Table I. (b) nanoDSC traces of the same samples and two additional mixtures measured at a scan speed of $2 \mathrm{~K} / \mathrm{min}$. previously reported model [11] shows that the micelles exhibit a typical core-shell structure consisting of a fully segregated, solvent-free core of constant density and an extended starlike PEO corona. The structural key parameters are given in Table I. Complementary SAXS data are shown in Fig. S1 in the Supplementary Material [12], together with the resulting fit parameters and a brief outline of the fit model. Evidently, the size of the 1:1 mixed micelles is in between those of the neat ones, indicating that both species mix on a molecular level. This is further supported by differential scanning calorimetry (nanoDSC) shown in Fig. 1(b). For mixed micelles, only a single exothermic peak is observed which is attributed to a cooperative melting process in the micellar core. The individual melting transitions widely disappear and therefore strongly suggest mixing of $\mathrm{C}_{28}$ and $\mathrm{C}_{22}$ blocks, even though the broadening of the transition indicates some local heterogeneities and a distribution of mobility caused by the small number of molecules present in the core and their tethering to the core-corona interface. The melting points and fusion enthalpies are also given in Table I. Noteworthily, the observed melting transitions are in quantitative agreement with density measurements performed on the same samples (see Fig. S2 in the Supplemental Material [12]). It is interesting that we observe miscibility of the $\mathrm{C}_{28}$ and $\mathrm{C}_{22}$ blocks $(\Delta n=6)$ in the core, even though bulk alkanes with $\Delta n>4$ are not miscible according to Kravchenko's rules $[13,14]$. This is consistent with studies reviewed by $\mathrm{Su}$ et al. [15] which show that micrometer confinement enhances the miscibility of $n$-alkanes. The enhanced miscibility is attributed to a suppressed lamellar ordering and longitudinal diffusion under confinement, which are processes involved in the phase separation of length-mismatching $n$-alkanes in bulk.

The kinetic experiments were performed by timeresolved SANS using a kinetic zero-average-contrast experiment [16]. Briefly, the method works by mixing two populations of micelles, one with a deuterated and the other with a proteated PEO block, both in a zero average contrast $\mathrm{H}_{2} \mathrm{O} / \mathrm{D}_{2} \mathrm{O}$ mixture $\left(\phi_{\mathrm{D}_{2} \mathrm{O}}=66.7 \mathrm{vol} \%\right)$, which matches exactly the average scattering length density of

TABLE I. Structural and calorimetric data of the investigated micelles obtained from fitting SANS curves and analyzing DSC traces, respectively: $N_{\text {agg }}$, aggregation number; $R_{m}$, micellar radius; $R_{c}$, core radius; $T_{m}$, melting point; $\Delta H_{\text {fus }}$, enthalpy of fusion.

\begin{tabular}{lrcccc}
\hline \hline & $N_{\text {agg }}$ & $R_{m}$ & $R_{c}$ & $T_{m}$ & $\Delta H_{\text {fus }}$ \\
\hline & & $(\AA)$ & $(\AA)$ & $\left({ }^{\circ} \mathrm{C}\right)$ & $(\mathrm{kJ} / \mathrm{mol})$ \\
\hline $\mathrm{C}_{28}-h$ PEO5 & $130 \pm 5$ & $135 \pm 5$ & $28 \pm 1$ & $57 \pm 1$ & $40 \pm 3$ \\
$3: 1$ & $108 \pm 5$ & $127 \pm 4$ & $26 \pm 1$ & $51 \pm 1$ & $39 \pm 3$ \\
$1: 1$ & $84 \pm 4$ & $121 \pm 4$ & $24 \pm 1$ & $43 \pm 1$ & $33 \pm 2$ \\
$1: 3$ & $72 \pm 3$ & $116 \pm 3$ & $22 \pm 1$ & $34 \pm 1$ & $27 \pm 1$ \\
$\mathrm{C}_{22}-h$ PEO5 & $57 \pm 2$ & $107 \pm 3$ & $20 \pm 1$ & $29 \pm 1$ & $25 \pm 1$ \\
\hline \hline
\end{tabular}


the two PEO blocks. We note that the deuterated polymers have almost the same PEO length as their proteated counterparts and that the deuteration has no significant effect on the micellar structure (as shown in the Supplemental Material of Ref. [4]). Upon mixing equal volumes of the oppositely labeled micellar solutions, initially at $t=0$, the two micelle populations scatter with maximum contrast. But as soon as molecules are exchanging, the contrast decreases to a minimum at $t=\infty$, where the $h$ - and $d \mathrm{PEO}$ chains are fully randomized and matched out by the solvent on larger length scales. Rapid mixing in the millisecond range was accomplished with a stoppedflow apparatus equipped with a separately temperaturecontrolled neutron probe head which ensures a high thermal stability in the range between 5 and $55^{\circ} \mathrm{C}$. From the integrated detector intensity $I(t)=\int I(Q, t) d Q$ the relaxation function $R(t)=\{[I(t)-I(\infty)] /[I(0)-I(\infty)]\}^{1 / 2}$ can be calculated [7], with the initial intensity immediately after mixing $I(0)$ and the intensity from randomly mixed micelles $I(\infty)$. This procedure allows for a much better time resolution (down to $25 \mathrm{~ms}$ ) compared to tracking the full scattering pattern $I(Q, t)$.

Example curves of $\mathrm{C}_{22}-\mathrm{PEO}, \mathrm{C}_{28}-\mathrm{PEO}$, and the $1: 1$ mixture at various temperatures are shown in Fig. 2. The neat micelles exhibit an exponential relaxation function, as predicted by the theory of Halperin and Alexander [3]. Surprisingly, the mixture shows two distinct exchange processes which can be modeled simply by a sum of two exponentials without any broadening. We intuitively attribute the faster process to $\mathrm{C}_{22}$ - and the slower process to $\mathrm{C}_{28}$-PEO5 molecules exchanging. That suggests that even though the different alkyl blocks mix inside the micellar core and crystallize cooperatively, the exchange processes are still decoupled, in agreement with previous reports on amorphous micelles $[8,9]$. This is shown more quantitatively in Fig. 3 which displays an Arrhenius plot for the

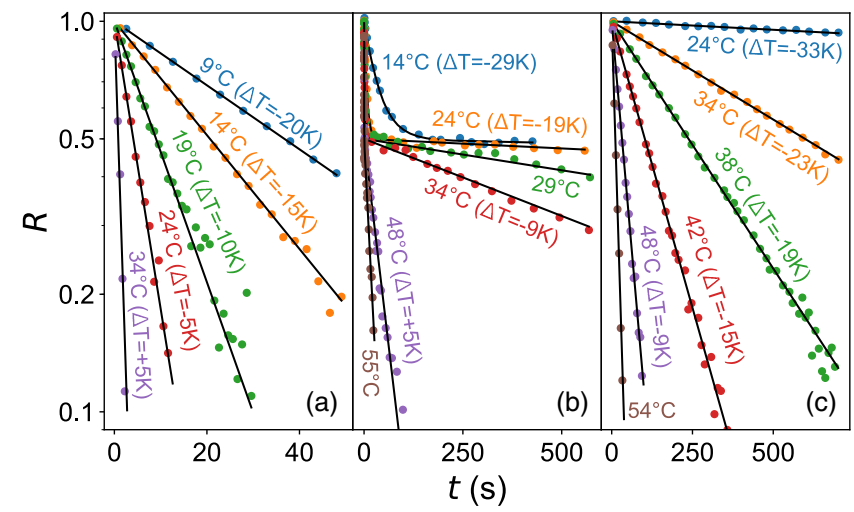

FIG. 2. Experimental relaxation functions and exponential fits at various temperatures. (a) $\mathrm{C}_{22}$-PEO5, (b) $\mathrm{C}_{28 / 22}$-PEO5 1:1, (c) $\mathrm{C}_{28}$-PEO5. The temperature difference $\Delta T=T_{\exp }-T_{m}$ to the melting point is given in brackets. Some curves have been left out for reasons of clarity. exchange times in the 1:1 mixture compared to those in the neat $\mathrm{C}_{n}$-PEO5 micelles. For an accurate comparison, a contour plot of the corresponding DSC traces, which shows the melting transition, has been included.

Evidently, two distinct regimes with different slopes emerge which reflect the molten and crystalline state as determined by nanoDSC. Moreover, the difference in slope in the Arrhenius plot, and hence, activation energy, exactly matches the enthalpy of fusion as determined from the nanoDSC experiments. For example, for neat $\mathrm{C}_{22}$-PEO5 we obtain $E_{a}=105 \mathrm{~kJ} / \mathrm{mol}$ below melting and $E_{a}=$ $80 \mathrm{~kJ} / \mathrm{mol}$ above melting, which corresponds very nicely with $\Delta H_{\text {fus }}=25 \mathrm{~kJ} / \mathrm{mol}$. Unfortunately, we could not compose a similar plot for neat $\mathrm{C}_{28}$-PEO5 because temperature stability in the stopped-flow setup was not achievable above the melting point $T_{m}=57^{\circ} \mathrm{C}$. Thus, the respective plot only exhibits a uniform slope below $T_{m}$ corresponding to the activation energy of $\mathrm{C}_{28}$-PEO5 in the crystalline regime $(170 \mathrm{~kJ} / \mathrm{mol})$. However, via reducing the melting point by mixing $\mathrm{C}_{28}$ - and $\mathrm{C}_{22}$-PEO5, we were able to obtain similar results for $\mathrm{C}_{28}-\mathrm{PEO}$. Analogous to neat $\mathrm{C}_{22}-\mathrm{PEO}$, the reduced melting point in the 1:1 mixture allows for an Arrhenius plot which is separated into the molten and crystalline regimes. Likewise, the activation energies of both regimes differ by the enthalpy of fusion. Additionally,

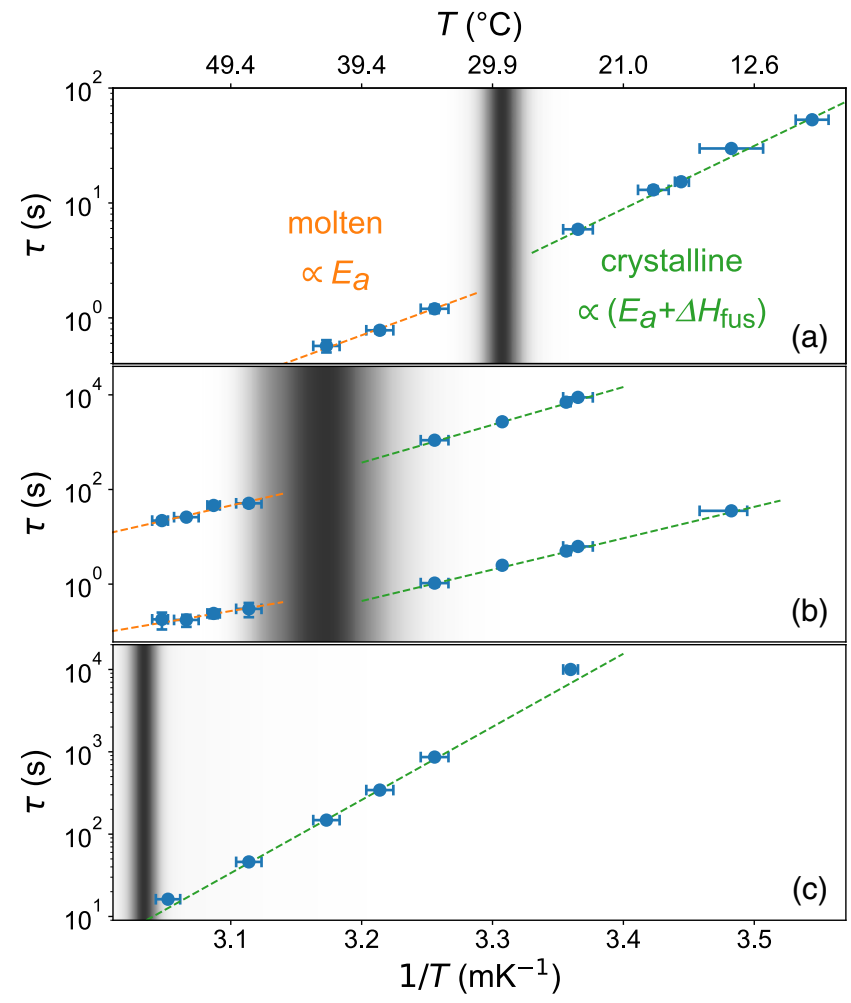

FIG. 3. Arrhenius plot of the temperature-dependent characteristic exchange times of (a) $\mathrm{C}_{22}$-PEO5, (b) the $\mathrm{C}_{28 / 22}$-PEO5 1:1 mixture, and (c) $\mathrm{C}_{28}$-PEO5. The gray shade is a contour plot of the respective nanoDSC melting curve and thus separates the molten and crystalline regimes. 
we performed a similar analysis of $1: 3$ and $3: 1$ mixtures (see Figs. S3 and S4 in the Supplemental Material [12]).

Based on these findings, activation energies obtained below $T_{m}$ have been corrected for the enthalpy of fusion, as measured by nanoDSC, via $E_{a} \rightarrow E_{a}-\Delta H_{\text {fus }}$. The thus obtained individual net activation energies $E_{a}$ of $\mathrm{C}_{22}-$ and $\mathrm{C}_{28}$-PEO5 in the different compositions are shown in Fig. 4(a). The plot clearly reveals that within experimental uncertainty, $E_{a}$ remains virtually unchanged for both alkane lengths in mixed micelles. Apparently, the individual expulsion of the alkane block is almost independent of the core composition, thus, confirming the "independent chain hypothesis" [9]. This is straightforwardly explained by the fact that-in the molten regimethe core blocks only "feel" an alkane environment, and the specific length of the neighboring chains does not significantly influence the energy barrier imposed by the additional hydrophobic surface upon chain expulsion. Nevertheless, when inspecting the $\mathrm{C}_{22}$-PEO5 activation energies more closely, there is a trend of increasing $E_{a}$ with increasing $\mathrm{C}_{28}$-PEO5 content. We attribute this to the influence of core-corona-interface area per chain as described by Zhao et al. [17]. As the average alkyl chain length in the mixture increases, so does the aggregation number and core radius (compare Table I), but the interface area per chain decreases $\propto 1 / R_{c}$. The "orifice" through which the alkyl block leaves the core thus becomes smaller and the corona denser, both effects hindering chain expulsion and increasing the apparent activation energy.
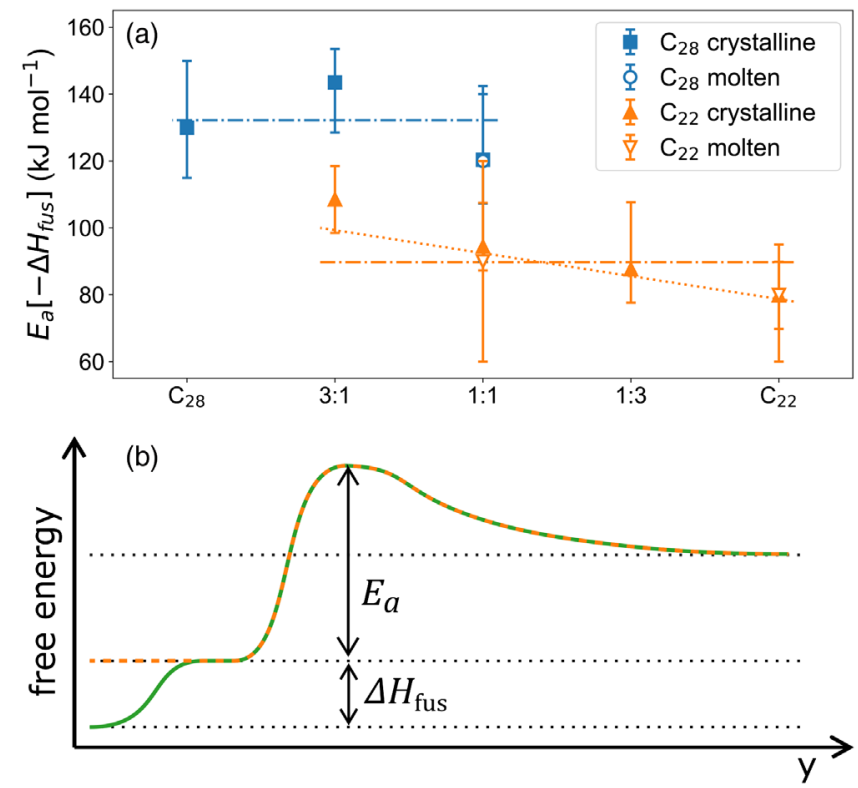

FIG. 4. (a) Activation energies of the chain expulsion step of $\mathrm{C}_{28}$ - and $\mathrm{C}_{22}$-PEO5 in various mixtures. Values obtained from the crystalline regime were corrected for the enthalpy of fusion determined from nanoDSC. The (dashed-)dotted lines are guides to the eye. (b) Tentative free energy landscape of the expulsion step where $y$ is the reaction coordinate.
We should note that the experimental uncertainty of the $\mathrm{C}_{28}$-PEO5 activation energies is generally larger than those of $\mathrm{C}_{22}$-PEO5, and hence prevent similar deductions for $\mathrm{C}_{28}$-PEO5. This is basically due to the necessary long collection times (several hours) to get the full relaxation profile and to determine accurately the final intensity, $I(\infty)$.

Inspecting the corresponding attempt times $\tau_{0}$ yields a similar picture (see Fig. S5 in the Supplemental Material [12]): When correcting for the entropy of fusion $\Delta S_{\text {fus }} \approx$ $\Delta H_{\text {fus }} / T_{m}\left[\tau_{0} \rightarrow \tau_{0} \exp \left(\Delta S_{\text {fus }} / R\right)\right.$, where $R$ is the universal gas constant] [4], the attempt times in the molten and crystalline regimes agree [18]. We thus propose that the cocrystallization gives an additional, cooperative energy barrier to the otherwise decoupled expulsion processes in mixed micelles as is depicted in Fig. 4(b).

To summarize, employing this well-defined model system, we show that cooperative solid-liquid phase transitions in nanocrystalline self-assembled micellar systems not necessarily imply cooperativity in intermicellar exchange processes. That implicates that the segmental diffusion (i.e., local conformational transitions and segmental motion) is essentially decoupled from the center-of-mass diffusion (i.e., molecular exchange). However, it should be noted that this does not translate to an effective friction coefficient but rather a modification of the activation energy of the expulsion step. Even though the expulsion process includes a partial premediating melting process as the chain escapes the crystalline core [19], we still find two unique release processes corresponding to each component in the binary mixtures. Furthermore, the data show that the activation energy of the expulsion step governing the diffusion process out of the core undergoes an abrupt reduction at the melting point that exactly amounts to the enthalpy of fusion. Very interestingly, the transition temperature can be accurately tuned by coassembling polymers with different $n$-alkyl length. Above the melting transition, this occurs without affecting the activation energies of the individual compounds, which only depend on the hydrophobic surface created upon chain expulsion. However, below the melting transition, an additional term $\Delta H_{\text {fus }}$ is added to the total activation energy, a quantity which depends on the mixing ratio and the nature of the $n$-alkane. Hence, cocrystallization, although less effective than varying the core block length [5,7], corona block length $[20,21]$, and molecular architecture [22-24], represents a handy tool to fine-tune the temperature response of the timescale on which the molecular exchange takes place. This is important insight for the accurate design of multicomponent self-assembling systems, although in the case of mixing chemically dissimilar components, additional modification of the dynamics may be expected, e.g., a change in the local segmental motion. We also shed light on the thermodynamics and kinetics of low-molecular-weight molecules in nanoconfined systems. It has been reported that confinement may induce demixing of small molecules like, for 
instance, water and methanol [25] or colloidal mixtures [26]. In our case, however, the data demonstrate that the spherical nanoconfinement greatly enhances the miscibility of crystalline long $n$-alkanes which are incompatible on a macroscopic scale-in agreement with previous studies on microconfinement [15]. Moreover, we show that the dynamics in crystalline self-assembled materials can be rather straightforwardly predicted from their amorphous state based on quantities easily measurable using standard techniques such as DSC. Similar ideas and experimental approaches can be applied within biophysics to understand the properties of membranes that consist of a range of lipids with similar chemical compositions but varying melting points.

This work is based upon experiments performed at the KWS-2 instrument [27] operated by JCNS at Heinz MaierLeibnitz Zentrum, Garching, Germany. We also acknowledge use of the Norwegian national infrastructure for x-ray diffraction and scattering (RECX). Furthermore, we thank Matthias Amann (Department of Chemistry, University of Oslo) for assistance during the neutron scattering experiments and Bente A. Breiby (Department of Pharmacy, University of Oslo) for performing the nanoDSC experiments.

*1.willner@fz-juelich.de

ORCID ID:0000-0001-6482-7937

${ }^{\dagger}$ Present address: ESRF—The European Synchrotron, 38043

Grenoble Cedex 9, France.

\#reidar.lund@kjemi.uio.no

ORCID ID:0000-0001-8017-6396

${ }^{8}$ ORCID ID:0000-0003-3319-5708

'ORCID ID:0000-0002-3930-3602

[1] J. J. Crassous, P. Schurtenberger, M. Ballauff, and A. M. Mihut, Design of block copolymer micelles via crystallization, Polymer 62, A1 (2015).

[2] T. Zinn, L. Willner, and R. Lund, Nanoscopic Confinement through Self-Assembly: Crystallization within Micellar Cores Exhibits Simple Gibbs-Thomson Behavior, Phys. Rev. Lett. 113, 238305 (2014).

[3] A. Halperin and S. Alexander, Polymeric micelles: their relaxation kinetics, Macromolecules 22, 2403 (1989).

[4] T. Zinn, L. Willner, V. Pipich, D. Richter, and R. Lund, Effect of core crystallization and conformational entropy on the molecular exchange kinetics of polymeric micelles, ACS Macro Lett. 4, 651 (2015).

[5] S. H. Choi, T. P. Lodge, and F. S. Bates, Mechanism of Molecular Exchange in Diblock Copolymer Micelles: Hypersensitivity to Core Chain Length, Phys. Rev. Lett. 104, 047802 (2010).

[6] R. Lund, L. Willner, J. Stellbrink, P. Lindner, and D. Richter, Erratum: Logarithmic Chain-Exchange Kinetics of Diblock Copolymer Micelles, Phys. Rev. Lett. 104, 049902 (2010).

[7] R. Lund, L. Willner, V. Pipich, I. Grillo, P. Lindner, J. Colmenero, and D. Richter, Equilibrium chain exchange kinetics of diblock copolymer micelles: effect of morphology, Macromolecules 44, 6145 (2011).

[8] J. Lu, S. Choi, F. S. Bates, and T. P. Lodge, Molecular exchange in diblock copolymer micelles: bimodal distribution in core-block molecular weights, ACS Macro Lett. 1, 982 (2012).

[9] J. Lu, F. S. Bates, and T. P. Lodge, Chain exchange in binary copolymer micelles at equilibrium: confirmation of the independent chain hypothesis, ACS Macro Lett. 2, 451 (2013).

[10] M. Kastantin, B. Ananthanarayanan, P. Karmali, E. Ruoslahti, and M. Tirrell, Effect of the lipid chain melting transition on the stability of DSPE-PEG(2000) micelles, Langmuir 25, 7279 (2009).

[11] T. Zinn, L. Willner, R. Lund, V. Pipich, M. S. Appavou, and D. Richter, Surfactant or block copolymer micelles? Structural properties of a series of well-defined $n$-alkyl-PEO micelles in water studied by SANS, Soft Matter 10, 5212 (2014).

[12] See Supplemental Material at http://link.aps.org/ supplemental/10.1103/PhysRevLett.122.078001 for additional experimental data and an outline of the treatment of scattering data.

[13] V. Kravchenko, The eutectics and solid solutions of paraffins, Acta Physicochim. URSS 21, 335 (1946).

[14] M. Dirand, M. Bouroukba, V. Chevallier, D. Petitjean, E. Behar, and V. Ruffier-Meray, Normal alkanes, multialkane synthetic model mixtures, and real petroleum waxes: Crystallographic structures, thermodynamic properties, and crystallization, J. Chem. Eng. Data 47, 115 (2002).

[15] Y. Su, G. Liu, B. Xie, D. Fu, and D. Wang, Crystallization features of normal alkanes in confined geometry, Acc. Chem. Res. 47, 192 (2014).

[16] L. Willner, A. Poppe, J. Allgaier, M. Monkenbusch, and D. Richter, Time-resolved SANS for the determination of unimer exchange kinetics in block copolymer micelles, Europhys. Lett. 55, 667 (2001).

[17] D. Zhao, Y. Ma, and T. P. Lodge, Exchange kinetics for a single block copolymer in micelles of two different sizes, Macromolecules 51, 2312 (2018).

[18] The different attempt times of $\mathrm{C}_{22}-$ and $\mathrm{C}_{28}-\mathrm{PEO}$ can be understood from the different gain in conformational entropy of the $n$-alkanes upon chain expulsion. This depends on the chain length $n$ as elaborated in Ref. [4].

[19] The hypothesis of crystallization inside the micellar core is based on the presented DSC and density measurements as well as wide-angle $\mathrm{x}$-ray scattering. However, a local (shortrange) order, which probably is indistinguishable from a crystalline order with very small correlation length, cannot be entirely excluded.

[20] T. Zinn, L. Willner, V. Pipich, D. Richter, and R. Lund, Molecular exchange kinetics of micelles: corona chain length dependence, ACS Macro Lett. 5, 884 (2016).

[21] Z. Li and E. E. Dormidontova, Equilibrium chain exchange kinetics in block copolymer micelle solutions by dissipative particle dynamics simulations, Soft Matter 7, 4179 (2011).

[22] A. Prhashanna and S.B. Chen, Chain exchange kinetics between linear ABA-type triblock copolymer micelles, Polymer 118, 22 (2017).

[23] J. Lu, F. S. Bates, and T. P. Lodge, Remarkable Effect of Molecular Architecture on Chain Exchange in Triblock Copolymer Micelles, Macromolecules 48, 2667 (2015). 
[24] A. J. Peters and T. P. Lodge, Chain exchange kinetics of asymmetric $\mathrm{B}_{1} \mathrm{AB}_{2}$ linear triblock and $\mathrm{AB}_{1} \mathrm{~B}_{2}$ branched triblock copolymers, Macromolecules 50, 6303 (2017).

[25] Y. Liu, S. Consta, and W. A. Goddard, Nanoimmiscibility: Selective absorption of liquid methanol-water mixtures in carbon nanotubes, J. Nanosci. Nanotechnol. 10, 3834 (2010).
[26] L. Wu, A. Malijevský, C. Avendaño, E. A. Müller, and G. Jackson, Demixing, surface nematization, and competing adsorption in binary mixtures of hard rods and hard spheres under confinement, J. Chem. Phys. 148, 164701 (2018).

[27] A. Radulescu, N. K. Szekely, and M.-S. Appavou, KWS-2: Small angle scattering diffractometer, J. Large-Scale Res. Facil. 1, A29 (2015). 